\title{
Accurate Measurement of Thermal Displacement in Filled Skutterudite by Scanning Transmission Electron Microscopy
}

\author{
Lijun Wu, Xiaoya Shi, Qiang Li and Yimei Zhu
}

Condensed Matter Physics and Materials Science Department, Brookhaven National Laboratory, Upton, New York, 11973

The efficiency of thermoelectric materials is characterized by the dimensionless thermoelectric figure of merit, $Z T=\alpha^{2} T / \rho \kappa$, where $\alpha$ is the Seebeck coefficient, $\rho$ the electrical resistivity, $T$ the temperature, and $\kappa$ the total thermal conductivity. To achieve high ZT, one needs to maximize the power factor $\alpha^{2} / \rho$, whereas minimize thermal conductivity $\kappa$. The unfilled skutterudites have a high power factor, but their thermal conductivity is moderately low, thus $Z T$ is small. High $Z T$ can be achieved in filled skutterudites by filling guest atoms into the cages formed by pnicogen atoms [1]. The filling atoms are loosely bound to the pnicogen atoms, leading to Einstein-like vibrational modes that significantly reduce the lattice thermal conductivity. Measurement of local disorder and lattice vibrations, especially thermal vibrations of the filled atoms is therefore important for understanding the underlying mechanisms of the reduced thermal conductivity in the filled skutterudites. Recently, we showed that thermal and static displacement in the layered thermoelectric $\mathrm{Ca}_{3} \mathrm{Co}_{4} \mathrm{O}_{9}$ can be determined accurately by simultaneous acquisition of high-angle-and mediate-angle- annular dark field images in scanning transmission electron microscopy (STEM) [2]. Here, we demonstrate that the thermal displacement in filled skutterudite can be measured by acquiring STEM images with annular dark field (ADF) and annular bright field (ABF) detectors simultaneously (Fig. 1a). Unlike diffraction analysis that derives the overall displacements in the crystal from the intensities of Bragg reflections, we directly measure the atomic displacement in real space, thereby enabling us to refine independently the atomic displacement of the filled atoms that is crucial to revealing their different nature in phonon scattering.

Among the different type of skutterudites, $\mathrm{CoSb}_{3}$ has attracted greatest interest [1]. $\mathrm{CoSb}_{3}$-based materials not only exhibit some of the best thermoelectric properties (large electrical conductivity and Seebeck coefficient), but also have abundant, less volatile, and less expensive chemical species than other alternative skutterudite materials. Skutterudite $\mathrm{CoSb}_{3}$ has a cubic structure with 8 corner sharing $\mathrm{CoSb}_{6}$ octahedra (Fig. 1b). The filled atoms (blue spheres) occupy the large cages among the $\mathrm{CoSb}_{6}$ octahedra. Fig. 1c and 1d show a pair of STEM images of $\mathrm{Yb} / \mathrm{Ga}$-filled $\mathrm{CoSb}_{3}$ simultaneously acquired by ADF and ABF detectors in aberration-corrected JEM-ARM200F microscope. Both images clearly show strong contrast of $\mathrm{Sb}$ as well as weak and diffuse contrast of filled $\mathrm{Yb} / \mathrm{Ga}$ atoms. To determine the effect of the thermal vibration of the filled atoms on the image contrast of ADF and ABF, we calculated a series of $\mathrm{ADF}$ and $\mathrm{ABF}$ images based on multislice method with frozen phonon approximation by changing the thermal displacement of the filled atoms (Fig. 2a and $2 b$ ). The intensity profiles from the calculated $\mathrm{ADF}$ and $\mathrm{ABF}$ images with different thermal mean-square displacement are shown in Fig. 2c and $2 \mathrm{~d}$. While the peak intensity of Sb columns is basically unchanged, the peak intensity and width of the $\mathrm{Yb} / \mathrm{Ga}$ column vary with the thermal displacement of the filled atoms. With the increase in the thermal displacement of the $\mathrm{Yb} / \mathrm{Ga}$, the $\mathrm{Yb} / \mathrm{Ga}$ peak intensity decreases for ADF image, but increases for $\mathrm{ABF}$ image. The peak width (full width at half maximum, $\mathrm{FWHM}$ ) of the $\mathrm{Yb} / \mathrm{Ga}$ column as a function of thermal mean-square displacement is plotted in Fig. 2e. It demonstrates that the increase in the peak width is more pronounced in $\mathrm{ABF}$ image than that in ADF image. Combined these unique 
features in $\mathrm{ADF}$ and $\mathrm{ABF}$ images, the thermal displacement of filled skutterudites can be accurately determined.

\section{References}

[1] Xun Shi, Jiong Yang, James R. Salvada, Miaofang Chi, Jung Y. Cho, Hsin Wang, Shengqiang Bai, Jihui Yang, Wenqing Zhang, and Lidong Chen, J. Am. Chem. Soc. 133, 7837 (2011).

[2] L. Wu, Q. Meng, Ch. Jooss, J. Zheng, H. Inada, D. Su, Q. Li and Y. Zhu, Adv. Funct. Mater. 23, $5728(2013)$.

[3] The work is supported by the U.S. DOE, under contract No. DE-AC02-98CH10886.
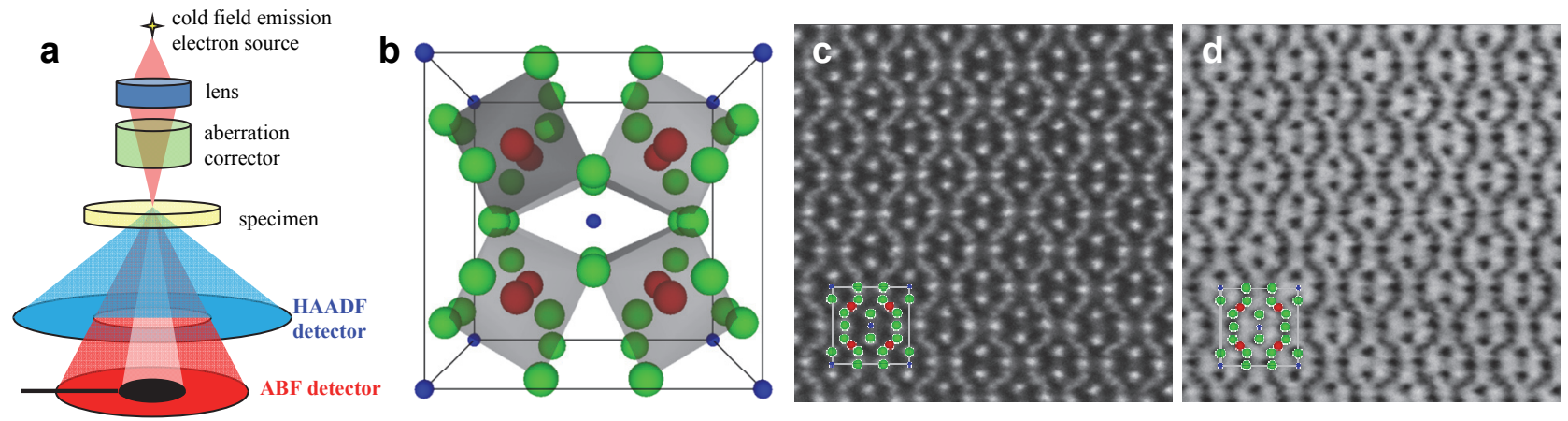

Fig. 1. (a) Experimental setup. (b) Structure model of the filled skutterudite $\mathrm{CoSb}_{3}$. The red, green and blue spheres represent $\mathrm{Co}, \mathrm{Sb}$ and filled $\mathrm{Yb} / \mathrm{Ga}$, respectively. (c,d) [001] STEM images of $\mathrm{Yb} / \mathrm{Ga}$-filled $\mathrm{CoSb}_{3}$ skutterudite simutaneously acquired by (c) annular dark field (ADF) and (d) annular bright field $(\mathrm{ABF})$ detectors. The [001] projection of the structure model is embedded in the images.
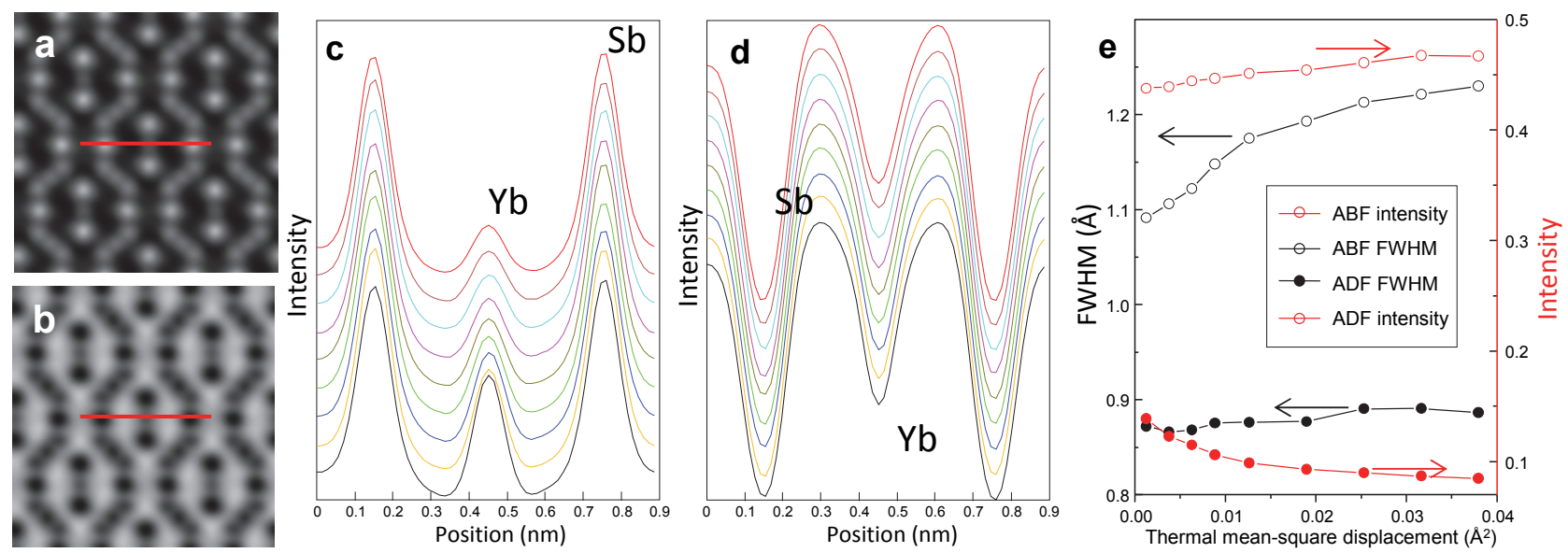

Fig. 2. (a,b) Calculated STEM images for (a) ADF and (b) ABF. (c,d) Intensity line profiles from a series of calculated (c) ADF and (d) ABF images from the position marked in (a) and (b). From the bottom line (black) to the top line (red), the thermal mean-square displacement increases from 0.001 $\AA^{2}$ to $0.038 \AA^{2}$. (e) Image intensity and full width at half maximum (FWHM) of ADF and ABF images for $\mathrm{Yb} / \mathrm{Ga}$ column as a function of thermal mean-square displacement. 